\title{
Switching from immediate- to sustained-release psychostimulants in routine treatment of children with attention-deficit hyperactivity disorder
}

\section{AIMS AND METHOD}

To investigate the effectiveness of switching children with attentiondeficit hyperactivity disorder (ADHD) from immediate- to sustainedrelease psychostimulants (Concerta $\mathrm{XL}$, a novel methylphenidate hydrochloride) and to examine factors associated with treatment success or failure. This was a retrospective study of all such children known to four clinicians in Lincolnshire, over a 2year period. The initial response to treatment and the response to slowrelease psychostimulant as judged by the clinicians were recorded. Data were analysed using the Statistical Package for the Social Sciences version 12.

\section{RESULTS}

Of the children who were switched $(n=97)$ and on whom clinical judgement was available $(n=92)$, a statistically significant number (32\%) responded poorly $(P<0.001)$. In 26 out of the 97 patients, the switching was considered as a treatment failure and they were switched back to the original immediate-release stimulants. There was no significantdifference with possible confounding variables between children who responded well and those who responded poorly after switching to sustained-release drug.

\section{CLINICAL IMPLICATIONS}

In a real-life clinical situation there is a significant failure rate when a child with ADHD is switched from an immediate- to sustained-release psychostimulant. Further studies are needed.
The psychostimulants methylphenidate and dexamphetamine have an established place in the management of childhood attention-deficit hyperactivity disorder (ADHD) (MTA Cooperative Group, 1999). Immediate-release methylphenidate has a short plasma half-life and typically three doses per day are required. Traditional sustainedrelease preparations of methylphenidate may last up to $8 \mathrm{~h}$ but are often not as effective as multiple doses of immediate-release preparations (Swanson et al, 1999) and have not been widely used in the UK. Concerta XL was the first once daily formulation of methylphenidate to be licensed in the UK, becoming available in February 2002. When ingested, a bolus of methylphenidate is delivered from its coating, followed by controlled delivery of the drug from the tablet's core using Osmotic Release Oral System (OROS) technology. This sustained-release preparation is said to have a similar onset and duration of efficacy as three times daily dosing of immediate-release methylphenidate.

\section{Method}

This is an observational and retrospective study where three child psychiatrists and one community paediatrician working in Lincolnshire provided information on their patients who were either currently taking or had had a trial of the novel sustained-release psychostimulant at any time between February 2002 and February 2004. The patients were identified by the four treating doctors using a combination of computer case-load databases and personal case-load records. This method was the best available but cannot be guaranteed to have produced a comprehensive list. A standardised pro forma was used to collect information about the patients, their response to immediate-release psychostimulants prior to switching, the reasons for change and the outcomes of treatment with the new drug. Results were collated and analysed using the Statistical Package for the Social Sciences (SPSS) version 12 for Windows.

\section{Results}

\section{Participants}

A total of 103 young people were identified: 86 boys and 17 girls, with a mean age of 12 years 9 months (range 6 17 years). The mean time since diagnosis was 4 years (range 7-108 months). Neither the duration of treatment with immediate-release psychostimulants prior to switching nor the titration process informing the dose of immediate-release psychostimulant was recorded. Six young people had been treated with the sustainedrelease preparation as the first-line drug in the management of their ADHD: these children were excluded from the study (Fig. 1).

\section{Reasons for switching}

Clinicians were asked to indicate why they had changed their patients' medication. More than one reason was given for each patient. The frequencies of different reasons given are shown in Fig. 2. The three most common reasons for switching were poor adherence to three times daily doses, erratic symptom control on immediate-release psychostimulants and a hope that patients would get a better therapeutic response. 


\section{Switching to an adequate dose}

original papers
To check that clinicians had switched children from immediate-release psychostimulants to an adequate dose of the sustained-release preparation, we looked at all the children whose initial dose of immediate-release psychostimulant corresponded to the doses of methylphenidate given in the manufacturer's dose algorithm (Janssen Cilag, 2002). There were 23 children initially taking the doses of immediate-release methylphenidate specified in the algorithm (methylphenidate $5 \mathrm{mg}$ three times daily, $10 \mathrm{mg}$ three times daily or $15 \mathrm{mg}$ three times daily). Twenty of these children had been switched to the dose of the sustained-release preparation recommended by the manufacturer, one child had been switched to a higher dose and only two had been switched to a lower than recommended dose. In addition, we compared doses of the new drug of those who had a good response to the switching (mean $41.4 \mathrm{mg}, \mathrm{s} . \mathrm{d} .=14.325$ ) with those who had poor response $(\mathrm{m} 39.1 \mathrm{mg}$, s.d. $=12.79)$. This difference was not statistically significant using either parametric $(t=0.779, P=0.439)$ or nonparametric tests (Mann-Whitney test; $P=0.554$ ) (the samples were not normally distributed). We concluded that clinicians were generally using an adequate dose of the sustained-release preparation.

\section{Outcomes after switching}

A judgement of the effectiveness of a trial of the sustained-release preparation could be made for 92 young people. Clinicians rated the response to immediate-release psychostimulants as 'minimally effective', 'moderately effective' or 'very effective'. They

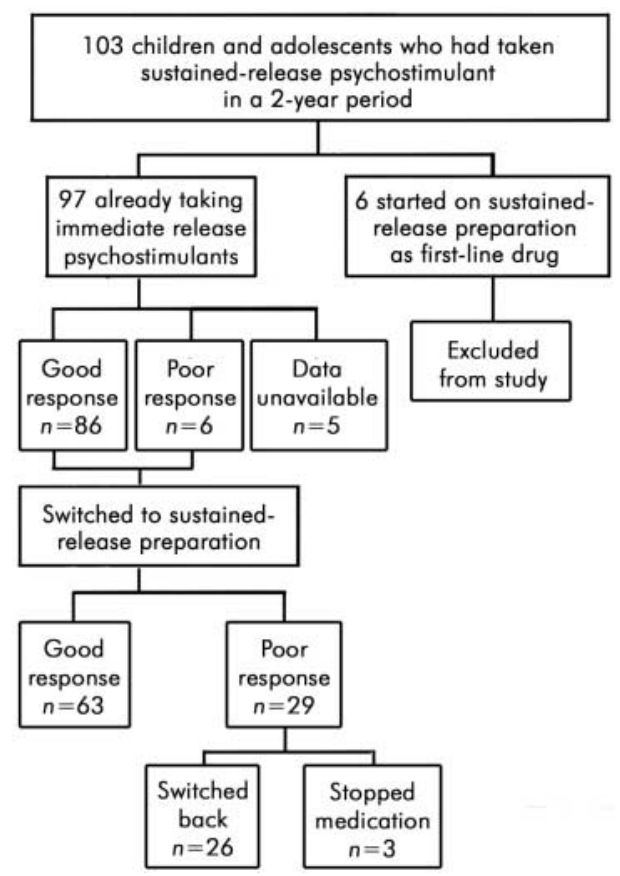

Fig. 1. Participants in the retrospective study of the new sustained-release psychostimulant (Concerta $X L$ ) were asked to rate the new treatment as 'better', 'same' or 'worse'. In all cases, the same clinician rated children on immediate-release psychostimulants and then when taking the new drug. Thirty-one children were rated as being 'better', 32 were rated as 'same' and 29 were rated as 'worse'.

For the analysis the 'better' and 'same' categories were combined to make a single category 'good'. Children rated as 'worse' were said to have a 'poor' outcome. All but one of them $(n=28)$ had previously had a 'moderately effective' or 'very effective' response to immediaterelease psychostimulants. The number of children with an adequate response to medication dropped from 86 on immediate-release psychostimulants to 63 on the new drug (Table 1). The outcome after switching was compared with the previous response using the McNemar test for paired nominal data. There was a significant decrease in good treatment response when children were switched $(P<0.001)$ (Table 1 and Fig. 3).

As the treatment outcome was a judgement made by the treating clinician without recourse to objective measurement, we analysed the outcome of switching in a second way. We assumed that if children and young people were switched back to immediate-release psychostimulants, then this indicated a clinically significant treatment failure. Twenty-six (27\%) children and young people were switched back to immediate-release psychostimulants. The switch back occurred in the first few months of treatment (mean time of the switch back was 2.7 months, range $0.5-6$ months). Manufacturer's data suggest that the sustained-release preparation is identical to three times daily immediate-release methylphenidate and so one could assume that the expected failure rate would be zero. Based on this assumption the $27 \%$ rate of switching back is statistically significant $(P<0.001)$ using binomial analysis. Even when we estimated that the expected treatment failure rate might be as high as 20\% (allowing for patients' dislike of a different preparation despite identical therapeutic equivalence) the statistical significance was maintained $(P=0.038)$

\section{Influence of possible confounding variables}

The characteristics of those children and young people who had a good response to the sustained-release preparation were compared with those who had a poor response. The tested variables were gender, age, time since diagnosis, erratic control on immediate-release psychostimulants, poor adherence to immediate-release psychostimulants and dose of new drug. Analysis using independent $t$-tests for continuous normally distributed variables, Mann-Whitney tests for continuous nonnormally distributed variables and $\chi^{2}$ tests for the other categorical variables failed to show any significant difference between poor responders and good responders (Table 2). 


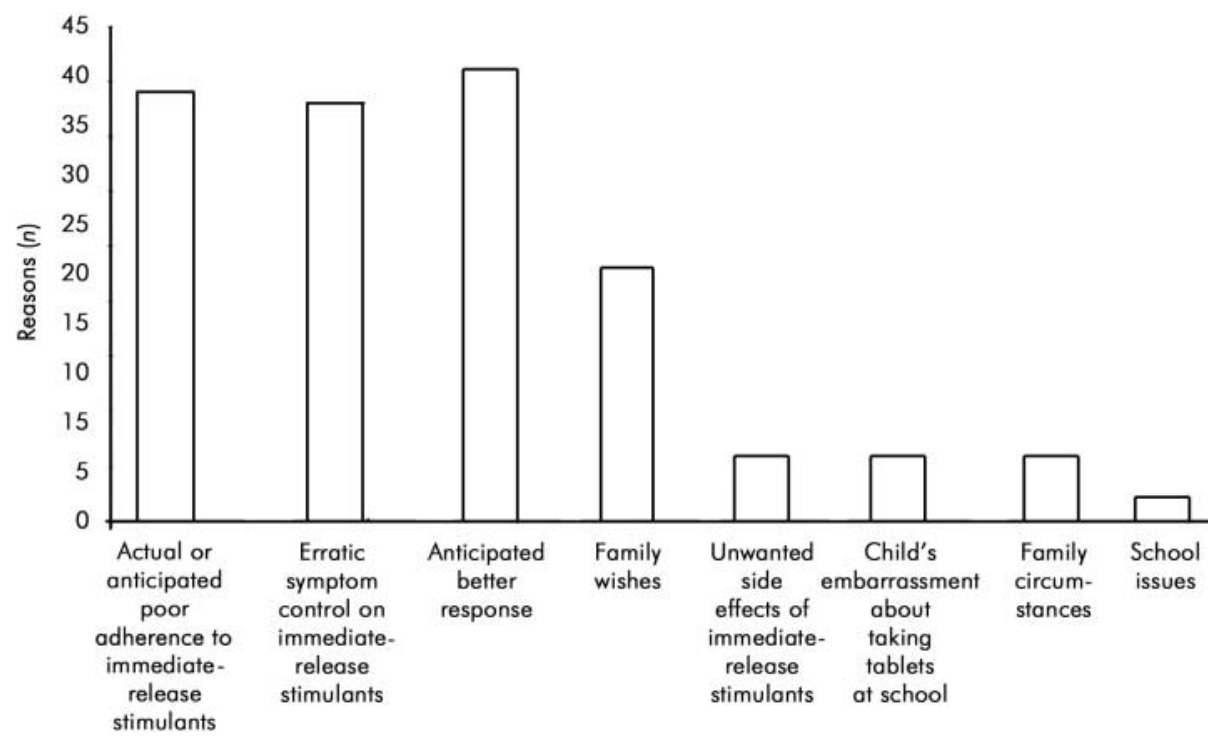

Fig. 2. Clinicians' reasons for switching to sustained-release preparation.

\section{Discussion}

The new sustained-release psychostimulant (Concerta $\mathrm{XL}$ ) has the same side-effect profile as immediate-release methylphenidate (Pelham et al, 2001). Its proposed advantage over immediate release methylphenidate is convenience and possible greater adherence to treatment (Rappley, 2001).

The results of this study of usual clinical care show that a significant proportion of young people (32\%) switched from immediate-release psychostimulants to the sustained-release preparation experience a poor treatment response. This failure rate is higher than the generally accepted failure rate of immediate-release methylphenidate in the treatment of ADHD (20\%). The discontinuation rate in this study is in fact lower than that reported by Hoare et al (2005) in their 12-month clinical trial $(47 \%)$. Our results are a reminder that clinicians should be prescribing the new sustained-release preparation with the knowledge that not all young people will benefit and some would do better on immediate-release psychostimulants.

The failure rate demonstrated in this study may reflect the tendency to use inadequate doses for some young people. Although the clinicians switched individuals on lower doses of immediate-release methylphenidate to appropriate doses of the sustained-release preparation according to the manufacturer's algorithm, anecdotal reports suggest some young people taking

Table 1. Treatment outcome on immediate release psychostimulants and after switching

\begin{tabular}{lrrr} 
Initial response to & \multicolumn{3}{c}{ Response after switching } \\
\cline { 2 - 4 } $\begin{array}{l}\text { immediate-release } \\
\text { stimulants }\end{array}$ & Poor & Good & Total \\
\hline Poor & 1 & 5 & 6 \\
Good & 28 & 58 & 86 \\
Total & 29 & 63 & 92 \\
\hline
\end{tabular}

higher doses of immediate-release methylphenidate may need to be switched to $72 \mathrm{mg}$.

The results of the Multimodal Treatment Study of Children with Attention-Deficit Hyperactivity Disorder showed the benefits of careful monitoring of stimulant medication on patient outcome. In our study, the number of clinical contacts were not measured or controlled for. However, we assume that young people switched to a new drug are more likely to be monitored closely by clinicians than those on established treatment regimes. Hence we suggest that the beneficial effect of more intensive monitoring would have been evident once patients had been switched. This potentially positive influence on outcome was not evident.

Although anecdotal reports suggest that many specialists in the UK treating children who have ADHD are now prescribing the new preparation, we have been unable to find any systematic descriptions of this practice. Drug trials have shown a discontinuation rate of $17 \%$ in an 8-week randomised open-label trial of OROS methylphenidate $v$. usual clinical care with immediate-release methylphenidate (Steele et al, 2006) and a 15\% discontinuation rate in a 3-week open label study (Hoare et al, 2005). When the study period of this second study was

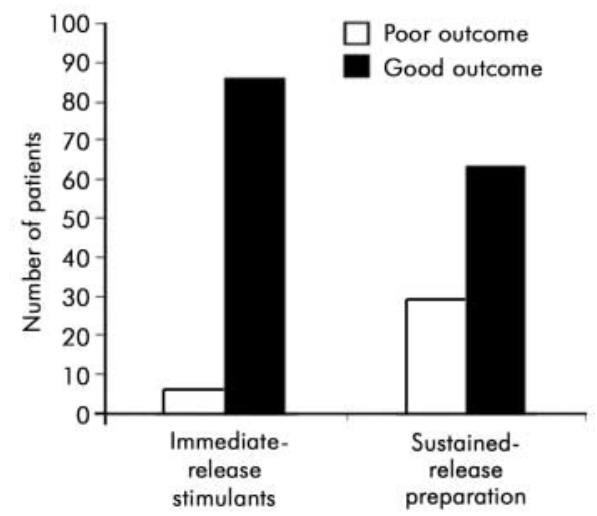

Fig. 3. Treatment outcome on immediate-release psychostimulants and after switching to Concerta XL. original papers 
Table 2. Significance of possible confounding variables on the outcome of switching

original

papers

\begin{tabular}{lccc}
\hline Variable & Poor outcome & Good outcome & $P$ \\
\hline Gender & & & \\
$\quad$ Male & 3 & 12 & 0.37 \\
Female & 26 & 51 & 0.24 \\
Age, years: mean (s.d.) & $12(3.22)$ & $13(2.50)$ & 0.32 \\
Time since diagnosis, months: mean (s.d.) & $51.7(23.8)$ & $48(29.0)$ & 0.50 \\
Erratic control on immediate-release psychostimulants & 13 & 23 & 0.36 \\
Poor adherence to immediate-release psychostimulants & 9 & 27 & 0.55 \\
Dose of sustained-release preparation, mg: mean (s.d.) & $39(12.7)$ & $41(14.3)$ & \\
\hline
\end{tabular}

extended to 12 months, the discontinuation rate rose to $47 \%$. Lack of efficacy was the main reason for discontinuation, with adverse events being the second most common reason. This clinical trial excluded some groups of children who are potentially hard to manage in clinical practice (e.g. children with psychiatric comorbidity and known non-responders to methylphenidate). In view of this bias, the fact that almost half of the sample discontinued treatment seems especially notable. However, the study was said to support the use of long-acting stimulants in preference to short-acting ones. The discontinuation rate of the new sustained-release preparation does not seem to be widely known.

Clinicians' views of 'benefit' were not precisely defined in our study. The group of children said to have benefited from the switch probably experienced a mixture of improved symptom control, better adherence and patient preference. These results are based only on information from clinicians. Clearly, the views of the young people, their families and teachers would add to the validity of these findings and give a broader picture.

Another limitation of this study is that improvement was measured according to the clinician's views and not an outcome scale. This was because of the study's retrospective nature. We suggest that in day-to-day clinical practice, decisions about treatment are strongly influenced by clinicians' views and so this measure of treatment success or failure has some face validity.

A prospective study including standardised outcome measurements and measures of patient adherence would add to our understanding of the place of the new sustained-release psychostimulant (Concerta $\mathrm{XL}$ ) in the management of childhood ADHD.

\section{Declaration of interest}

None.

\section{References}

HOARE, P., REMSCHMIDT, H. \& MEDORI, R. et al (2005) 12-month efficacy and safety of OROS MPH in children and adolescents with attention-deficit hyperactivity disorder switched from $\mathrm{MPH}$. European Journal of Child and Adolescent Psychiatry, 14, 305-309.

MTA COOPERATIVE GROUP (1999) A 14-month randomized clinical trial of treatment strategies for attentiondeficit/hyperactivity disorder. Archives of General Psychiatry, 56, 1073-1086.

PELHAM, W., GNAGY, E., BURROWSMACLEAN, L., et al (2001) Once-a-day Concerta methylphenidate versus three-times-daily methylphenidate in laboratory and natural settings. Pediatrics, 107, 105.

RAPPLEY, M. (2001) Methylphenidate (OROS ${ }^{\circledR}$ formulation). CNS Drugs, 15, 501.

STEELE, M., WEISS, M., SWANSON, J., et al (2006) A randomized, controlled, effectiveness trial of OROSmethylphenidate compared to usual care with immediate-release methylphenidate in attention-deficit hyperactivity disorder. Canadian Journal of Clinical Pharmacology, 13 e50-e62.

SWANSON, J. GUPTA, S., GUINTA, D., et al (1999) Acute tolerance to methylphenidate in the treatment of attention deficit hyperactivity disorder in children. Clinical Pharmacology and Therapeutics, 66, 295-305.

*Anne E.Thompson Consultant Child and Adolescent Psychiatrist, Lincolnshire Partnership NHS Trust, Child and Family Services, Moore House, 10/11 Lindum Terrace, Lincoln, LN2 5RS, e-mail: Annelizthompson@aol.com, Saeed A. Nazir Consultant Child and Adolescent Psychiatrist, Lincolnshire Partnership NHS Trust, Grantham Health Clinic, Grantham, Mohammed J. Abbas Senior House Officer in Psychiatry, Lincolnshire Partnership NHS Trust, Moore House, Lincoln, Julie Clarke Associate Specialist in Community Paediatrics, United Lincolnshire HospitalsTrust, Grantham and District General Hospital, Grantham 